\title{
Enhanced FAD Production in Eremothecium ashbyi with Statistically Optimized Oxidative Stress Modulators
}

\author{
Manan Vipulbhai Patel, Chandra Sainathan* \\ Department of Biotechnology, Indian Institute of Technology Madras, Chennai, India \\ Email address: \\ manan2349@gmail.com (M. V. Patel), chandra@iitm.ac.in(C. Sainathan) \\ To cite this article: \\ Manan Vipulbhai Patel, Chandra Sainathan. Enhanced FAD Production in Eremothecium ashbyi with Statistically Optimized Oxidative Stress \\ Modulators. International Journal of Microbiology and Biotechnology. Vol. 5, No. 1, 2020, pp. 7-15. doi: 10.11648/j.ijmb.20200501.12
}

Received: December 12, 2019; Accepted: December 27, 2019; Published: January 31, 2020

\begin{abstract}
Riboflavin (vitamin B2) and flavin adenine dinucleotide (FAD) are valuable biological flavins mainly used as animal feed supplement and in pharmaceutical formulation. Eremothecium ashbyi is a natural overproducer of FAD along with riboflavin. Flavins overproduction was found to be strongly correlated with stress induction but stress response in E.ashbyi is completely unexplored. Hence, in the present study, flavins production in presence of four oxidative stress modulators (menadione, vitamin E, cAMP, $\mathrm{H}_{2} \mathrm{O}_{2}$ ) was investigated. Their levels were statistically optimized to maximize the flavins production. The regression model obtained from Central Composite Design indicated that maximum flavins production occurred when $1.09 \mu \mathrm{M}$ menadione and $1.12 \mu \mathrm{M}$ vitamin $\mathrm{E}$ were supplemented to 24-hour grown fungus. Upon supplementation, total flavins production was significantly increased by 1.34 -fold and FAD production was increased by 2.18 fold over untreated control. Intracellular - reactive oxygen species (ROS) level was increased and reduced glutathione (GSH) and oxidized glutathione (GSSG) ratio was decreased indicating oxidative burst experienced by the cell. Furthermore, a concomitant increase in specific activity of glutathione reductase and FAD synthetase was observed. For the first time this study showed that among flavins, FAD was majorly increased along with FAD synthetase activity as a response to oxidative stress. These results indicated that the oxidative stress and flavins overproduction was centered on FAD regulation.
\end{abstract}

Keywords: Eremothecium ashbyi, Riboflavin, FAD, Central Composite Design (CCD), Glutathione, Oxidative Stress

\section{Introduction}

Flavins are important biological compounds with common isoalloxazine moiety of tri-heterocyclic structure called flavin ring. Riboflavin, FMN and FAD are major flavins involved in several metabolic reactions vital for life. Riboflavin, also known as vitamin $\mathrm{B}_{2}$, is used as food and feed additive with nearly 10000 tons of production per year. FMN and FAD are co-enzymes for important oxidation/reduction metabolic reactions [1]. FAD has a pharmaceuticals application and 1000 times more expensive than riboflavin. It is used as an ophthalmic agent and multi-vitamin supplements. [2]. FAD is used in the treatment of genetically inherited diseases like Friedreich ataxia which is caused by the lack of the mitochondrial protein frataxin [3] and chronic granulomatosis which is caused by mutations in leukocyte NADPH oxidase genes [4].

Eremothecium ashbyi is a filamentous fungus belonging to Saccharomycetaceae family [5]. It is a natural overproducer of riboflavin and was used for industrial riboflavin production [6]. A closely related fungus, Ashbya gossypii was preferred to E.ashbyi for industrial production of riboflavin as E.ashbyi was genetically unstable. Moreover, annotated genome sequence database as well as genetic engineering tools were developed for A.gossypii [7]. However, E.ashbyi in contrast to A.gossypii is reported to overproduce FAD [8] and this property has not been exploited so far.

The basis for over production of flavins in stationary to lysis stage by these filamentous fungi is still intriguing. Riboflavin production as response to stress has been well explored in A.gossypii and Pichia guilliermondii [9]. Amongst the different types of stress tested [10], oxidative stress strongly correlated with riboflavin production in A.gossypii [11]. cAMP supplementation which generates stress relieving signals, decreased the riboflavin production [12] while oxidative stress inducers like hydrogen peroxide and menadione increased it in A.gossypii [13]. Stress 
response transcription factor Yap-1 mediated increase in riboflavin production was observed upon supplementation of hydrogen peroxide in A.gossypii. This provided a gene-level association between riboflavin production and oxidative stress [14]. Thus, flavins emerged as a group of potential anti-oxidants whose effects are synergistically attributed through mainly glutathione redox cycle [15]. The potential of E.ashbyi for production of pharmaceutically important metabolite FAD upon oxidative stress through a combination of stress modulators has not been explored.

In this study, the effect of oxidative stress modulators on the production of the flavins was studied for the first time in E.ashbyi. The effect of combination of five different factors on flavins production in E.ashbyi was statistically analyzed. The significant factors' levels were optimized through Central Composite Design and regression analysis. Since glutathione is a key regulator of oxidative stress, ratio of reduced to oxidized glutathione (GSH/GSSG) and activity of stress response enzyme glutathione reductase were determined. Glutathione reductase requires FAD to convert oxidized glutathione to its active reduced form. Thus, glutathione metabolism could be correlated to increased FAD levels upon oxidative stress.

\section{Materials and Methods}

\subsection{Strain and Growth Conditions}

Eremothecium ashbyi strain-NRRL Y-1363 was maintained on yeast extract-malt extract-broth (YM brothyeast extract- $3 \mathrm{gm}$, malt extract- $3 \mathrm{gm}$, peptone- $5 \mathrm{gm}$, glucose-10 gm per liter). A 48 -hour pre-inoculum of $0.5 \%$, grown on YM-broth at $180 \mathrm{rpm}$ at $30^{\circ} \mathrm{C}$, was used to inoculate $50 \mathrm{ml} \mathrm{YM}$ broth prepared in $250 \mathrm{ml}$ brown colored Erlenmeyer conical flask for all experiments.

\subsection{Oxidative Stress Modulators Addition to YM Broth}

Stock solutions of the following were prepared as follows.

a) Vitamin E (Sigma) (MW: 472.8 gm): A stock solution of $10 \mathrm{mM}$ was made in absolute ethanol. $1 \mathrm{mM}$ working stock was made from $10 \mathrm{mM}$ stock in absolute ethanol.

b) Dibutryl cAMP (SRL) (MW: 491.37 gm): A stock solution of $50 \mathrm{mM}$ was prepared in sterile water.

c) Menadione (Sigma) (MW: 376.23 gm): A stock solution of $10 \mathrm{mM}$ stock was prepared in sterile water.

d) Hydrogen peroxide (Merck) $\left(\mathrm{H}_{2} \mathrm{O}_{2}\right)-30 \% \mathrm{w} / \mathrm{v}$ stock was diluted to desired concentration just before use.

All stock solutions were filter sterilized with $0.2 \mu$ m nylon syringe filters.

\subsection{Estimation of Biomass and Residual Glucose}

Fungal mycelium was harvested by filtering through Whatman $^{\circledR}$ grade No. 1 filter paper followed by multiple washing and drying at $90^{\circ} \mathrm{C}$ until constant weight was obtained. Residual glucose was estimated using 3, 5Dinitrosalicylic acid (DNS) method.

Estimation of Flavins and FAD.
Total flavins and extracellular flavins were measured fluorometrically using the ISI-standard procedure (IS: 5399 1969) as described by Kavitha and Chandra, 2009 [11]. Briefly, extracellular flavins were measured by filtering the broth through Whatman $\left.{ }^{(}\right)$grade No. 1 filter paper. To estimate total flavins, broth was treated with four volumes of $0.02 \mathrm{~N} \mathrm{HCl}$, autoclaved at $121^{\circ} \mathrm{C}$ to break vacuoles and release intracellular flavins in supernatant and filtered to remove cell debris. Individual flavins - riboflavin, FMN and FAD were estimated as per Patel and Chandra, 2019 [16], through reverse phase HPLC gradient elution with column: HiQ Sil C18HS $(4.6 \mathrm{~mm} \times 250 \mathrm{~mm}, 5 \mu \mathrm{m})$. For total FAD estimation, the samples were autoclaved as mentioned earlier and treated with $10 \%$ Trichloroacetic acid (TCA) to remove proteins before injecting in HPLC.

\subsection{Preparation of Cell-Free Extracts (CFE)}

The cell-free extract was made by the method of AyarKayali et al., 2002 [17]. A constant amount of washed E.ashbyi mycelium was frozen at $-20^{\circ} \mathrm{C}$. The cells were suspended in $20 \mathrm{mM}$ sodium phosphate buffer, $\mathrm{pH} 7.0$ and grinded with sterile acid-washed glass beads in a mortar for $15 \mathrm{~min}$ over ice bath. The total volume was made up to $5 \mathrm{ml}$ with sodium phosphate buffer and centrifuged at 10,000 rpm for $10 \mathrm{~min}$ to remove cell debris. The protein content in the extract was determined by Bradford method.

\subsection{Reduced and Total Glutathione Estimation in CFE}

The reduced glutathione was estimated as per Tietze, 1969 [18]. 5, 5'-dithiobisnitrobenzoic acid (DTNB) reacts with reduced glutathione to form the colored product 5-thio-2nitrobenzoic acid (TNB) which is measured at $412 \mathrm{~nm}$. CFE (containing $0.5-1.0 \mathrm{mg}$ of protein $/ \mathrm{ml}$ ) was treated with $10 \%$ TCA to remove the proteins. $1 \mathrm{ml}$ assay reaction mixture contained $200 \mathrm{mM}$ sodium phosphate buffer, $\mathrm{pH}-8,6 \mathrm{mM}$ DTNB in phosphate buffer and treated CFE. The reaction mixture was incubated for 10 minutes and colour developed was determined at $412 \mathrm{~nm}$. The reduced glutathione was expressed as nmoles/mg of protein.

For measuring total glutathione, the oxidized glutathione (GSSG) present in the sample was converted to GSH by the highly specific glutathione reductase (Sigma) and NADPH (Sigma). The formation of the yellow colored compound TNB upon reaction of GSH with DTNB was measured at 412 $\mathrm{nm}$ as stated earlier. Reaction mixture contained $200 \mathrm{mM}$ sodium phosphate buffer $\mathrm{pH}-8$ (with $0.248 \mathrm{mg} / \mathrm{ml}$ of NADPH), $6 \mathrm{mM}$ DTNB in phosphate buffer, $5 \mu \mathrm{l}$ of 266 $\mathrm{U} / \mathrm{ml}$ of glutathione reductase and treated CFE.

\subsection{Glutathione Reductase (GR) Assay}

The assay was performed as per Mavis and Stellwagen, 1968 [19]. Glutathione reductase catalyzes the NADPH dependent reduction of glutathione disulfide (GSSG) to glutathione (GSH). The oxidation of NADPH to NADP is accompanied by a decrease in absorbance at $340 \mathrm{~nm}$. One Unit (U) of the enzyme was defined as $1.0 \mu$ mole of NADPH 
oxidized per minute at $25{ }^{\circ} \mathrm{C}$. at $\mathrm{pH}$ 7.0. The total reaction contained $-100 \mathrm{mM}$ potassium phosphate buffer $\mathrm{pH}-7,0.2$ $\mathrm{mM}$ GSSG, $0.02 \mathrm{mM}$ of NADPH and CFE (0.1-0.2 $\mathrm{mg}$ of protein $/ \mathrm{ml})$.

\subsection{FAD Synthetase Assay}

The assay was performed as per Yatsyshyn et. al., 2014 [2]. The assay mixture contained $75 \mathrm{mM}$ potassium phosphate buffer (pH -7.5), $1.5 \mathrm{mM} \mathrm{MgCl} 2,0.065 \mathrm{mM}$ FMN and $2 \mathrm{mM}$ ATP. The reaction mixture was incubated at $30^{\circ} \mathrm{C}$ for 30 minutes. The reaction was stopped by incubating the assay mix at $65^{\circ} \mathrm{C}$ for $10 \mathrm{~min}$ and immediately transferring to the ice. The FAD produced is quantified with reverse phase HPLC as stated earlier. One Unit (U) of enzyme activity was defined as $1.0 \mu$ mole of FAD produced per minute at $30^{\circ} \mathrm{C}$ at $\mathrm{pH} 7.5$.

\subsection{Reactive Oxygen Species (ROS) Assay}

Intracellular ROS was assayed by slightly modifying the method given by Rastogi et. al. 2010 [20]. 2',7 - dichloro fluoresce in diacetate (DCFDA) is a fluorogenic dye which can diffuse through cell membrane and gets deacetylated by cellular esterase which is then oxidized to fluorescent compound 2', 7' -dichloro fluorescein (DCF) by ROS. The DCF can be quantified by spectro-fluorimeter with excitation wavelength of $495 \mathrm{~nm}$ and emission wavelength of $529 \mathrm{~nm}$ after quenching with $10 \mathrm{mg} / \mathrm{ml}$ of sodium dithionite. The mycelium was harvested after exposing to oxidative stress modulators - menadione and vitamin E for 6 hours. The assay mixture for ROS determination contained $100 \mathrm{mg}$ of washed mycelium in phosphate buffer saline (PBS) with $5.0 \mu \mathrm{M}$ of DCFDA. ROS levels are reported indirectly as amount of $\mathrm{DCF}$ in nmoles/mg of protein.

\subsection{Factorial Design of Experiment and Response Surface Methodology}

Factorial design of experiments was carried out to identify important oxidative stress modulating factors and interaction among them. Five factors were considered: A.) Menadionesuperoxide radical generator. B.) $\mathrm{H}_{2} \mathrm{O}_{2}$-peroxide radical generator $\mathrm{C}$.) Vitamin $\mathrm{E}$ an antioxidant and a pro-oxidant in rare cases D.) Dibutryl-cAMP - negative stress regulator E.) Incubation time ie. time of supplementation of stress modulators post-inoculation. Extracellular flavins, total flavins and percentage excretion were recorded as responses in 120-hour grown culture. Half factorial design with 5 factors and level 5 resolution was used to screen significant factor for above mentioned responses. The limits of each variable were chosen from literature data and preliminary investigations carried out with individual factor. As the design level 5 resolution, effect of individual factor was confounded with four factor interactions and two factor interaction effect was confounded with three factor interactions. The data was analyzed with MINITAB 14 software. The factors significant at $95 \%$ confidence interval $(\mathrm{p}<0.05)$ were considered significant for total flavins production.

Experiments for optimization by Central Composite Design (CCD) were performed with the two most significant factors. Central composite design which is widely used response surface optimization strategy was employed to optimize supplement concentration of menadione and vitamin E. Both the factors' levels were decided from the above two runs of factorial design. Both factors were studied at five different levels (coded units: $-1,+1$ (corner point), $1.414,+1.414$ (star points) and 0 (center point). Contour plots and surface response plots were drawn using MINITAB 14. Multiple regression analysis was done on the experimental data to obtain a model with high $\mathrm{R}^{2}$ (multiple coefficient of regression) and low p-value. The model was then used to predict maximum flavins production. The model's prediction was checked and the model was validated.

\section{Results and Discussion}

\subsection{Effect of Individual Stress Modulators on Flavins Production}

To analyse the preliminary response of E.ashbyi against oxidative stress, the growth media was individually supplemented with three different concentration of $\mathrm{H}_{2} \mathrm{O}_{2}$ (1.0, 2.5 and $5.0 \mathrm{mM})$, menadione $(0.5,1.0$ and $2.5 \mu \mathrm{M})$ and vitamin $\mathrm{E}(0.5,1.0$ and $2.5 \mu \mathrm{M})$. The total flavins were estimated at 72 and 120-hour of growth. Maximum total flavins and FAD production was obtained with $1.0 \mu \mathrm{M}$ menadione at 120-hour compared to untreated control (Table 1). FAD was also increased by 1.21 -fold with $1.0 \mu \mathrm{M}$ vitamin E and 1.35-fold with $1.0 \mu \mathrm{M}$ menadione at 120-hour (Table 1). Vitamin $\mathrm{E}$ and $\mathrm{H}_{2} \mathrm{O}_{2}$ supplement had no significant effect on total flavins production and $5 \mathrm{mM} \mathrm{H}_{2} \mathrm{O}_{2}$ level was toxic. A.gossypii was reported to significantly increase flavins production with $\mathrm{H}_{2} \mathrm{O}_{2}$ [13]. This indicated that, although A.gossypii and E.ashbyi are closely related species, they responded quite differently to oxidative stress. It was of interest to study combined effect of oxidative stress supplements as they may act synergistically to enhance flavins rather than individual supplement by plausible activation of multiple pathways leading to enhanced expression of flavin genes.

Table 1. Effect of individual oxidative stress modulators on flavins production.

\begin{tabular}{llll}
\hline Sample & Total Flavins 72-hour $(\mathbf{m g} / \mathbf{L})$ & Total Flavins 120-hour $(\mathbf{m g} / \mathbf{L})$ & Total FAD 120-hour $(\mathbf{m g} / \mathbf{L})$ \\
\hline Control & $49.75 \pm 2.15$ & $118.01 \pm 3.00$ & $13.63 \pm 0.79$ \\
$\mathrm{H}_{2} \mathrm{O}_{2}$ & & & \\
$1.0 \mathrm{mM}$ & $52.6 \pm 2.30$ & $128.38 \pm 2.87$ & - \\
$2.5 \mathrm{mM}$ & $55.25 \pm 2.35$ & $130.26 \pm 5.00$ & $16.13 \pm 0.32 *$ \\
$5.0 \mathrm{mM}$ & $40.1 \pm 1.30$ & $106.51 \pm 2.50$ & - \\
\hline
\end{tabular}




\begin{tabular}{llll}
\hline Sample & \multicolumn{1}{l}{ Total Flavins 72-hour $\mathbf{( m g / L )}$} & Total Flavins 120-hour $(\mathbf{m g} / \mathbf{L})$ & Total FAD 120-hour $(\mathbf{m g} / \mathbf{L})$ \\
\hline Menadione & & & - \\
$0.5 \mu \mathrm{M}$ & $56.25 \pm 1.24$ & $129.13 \pm 3.38$ & $18.40 \pm 0.81^{*}$ \\
$1.0 \mu \mathrm{M}$ & $56.85 \pm 1.15$ & $137.88 \pm 4.13^{*}$ & - \\
$2.5 \mu \mathrm{M}$ & $51.9 \pm 1.10$ & $127.76 \pm 3.75$ & - \\
Vitamin E & & & $16.49 \pm 0.53^{*}$ \\
$0.5 \mu \mathrm{M}$ & $53.05 \pm 1.82$ & $127.12 \pm 1.50$ & - \\
$1.0 \mu \mathrm{M}$ & $64.04 \pm 1.19^{*}$ & $129.18 \pm 0.92$ & $123.59 \pm 2.01$ \\
$2.5 \mu \mathrm{M}$ & $60.91 \pm 1.45$ & & \\
\hline
\end{tabular}

Control $=$ Untreated culture, $*=p$-value $<0.05$.

\subsection{Screening of Combination of Stress Modulators Affecting Flavins Production}

Factorial experimental design was made with MINITAB 14 with five different factors and extracellular as well as total flavins and percentage excretion were recorded as responses (Table 2). Pareto chart obtained from the experiment showed menadione, vitamin $\mathrm{E}$ and time of supplementation to be most significant to increase the total flavins production (Figure 1). cAMP, a well-known negative stress regulator significantly decreased the total flavins production suggesting oxidative stress indeed played an important role in modifying regulation on flavins synthesis in E.ashbyi. cAMP was also reported previously to suppress riboflavin production in A.gossypii [12].
Menadione is most significantly affecting the total flavins production followed by vitamin E (Figure 1A). Vitamin E had profound effect on flavins excretion which could be observed when percentage excretion was recorded as response (Figure 1B). Vitamin $\mathrm{E}$ was also reported to increase the excretion in closely related fungus Ashbya gossypii [11]. Increased extracellular flavins is a desirable property due to ease and decreased loss during product recovery. Similar to A.gossypii, E.ashbyi also seems to respond with very low concentration of vitamin $\mathrm{E}$ compared to Ustilago maydis which was supplemented with $232 \mu \mathrm{M}$ vitamin $\mathrm{E}$ to be protected from fungicide toxicity [21]. Adjusted $\mathrm{R}^{2}-$ value for obtained factorial DOE model was 0.637 .

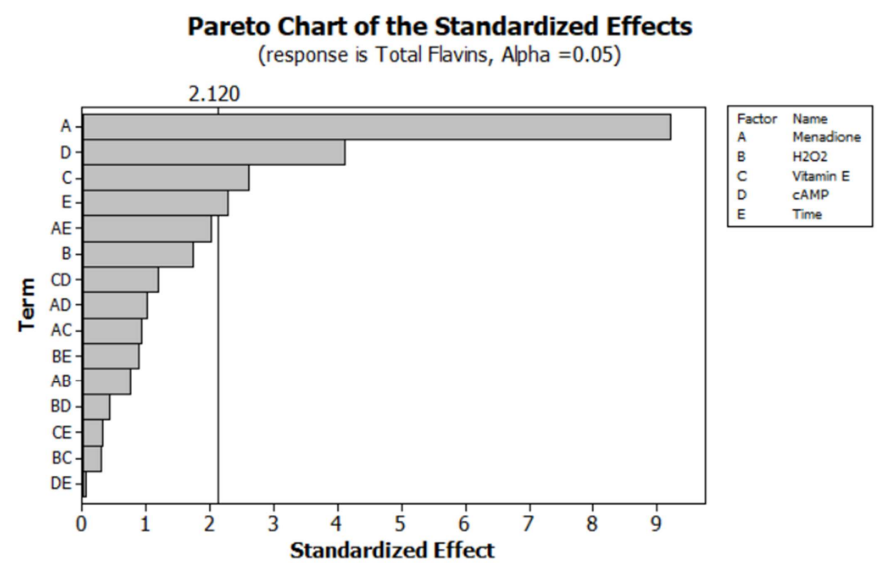

(A).

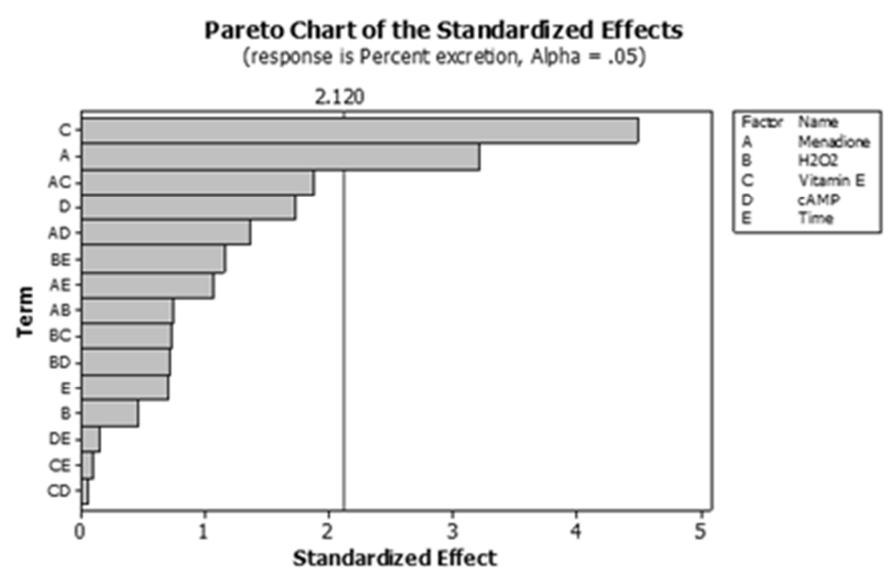

(B).

Figure 1. Factorial design of experiments for screening significant factors affecting flavins production. Pareto charts for standardized effects on A.) Total flavins production. B.) Percentage excretion. 
Table 2. Factorial design of experiment.

\begin{tabular}{|c|c|c|c|c|c|c|c|c|}
\hline $\begin{array}{l}\text { Run } \\
\text { Order }\end{array}$ & $\begin{array}{l}\text { Menadione } \\
(\mu \mathrm{M})(\mathrm{A})\end{array}$ & $\begin{array}{l}\mathrm{H}_{2} \mathrm{O}_{2} \\
(\mathrm{mM})(\mathrm{B})\end{array}$ & $\begin{array}{l}\text { Vitamin E } \\
(\mu \mathrm{M})(\mathrm{C})\end{array}$ & $\begin{array}{l}\text { cAMP } \\
(\mu \mathrm{M})(\mathrm{D})\end{array}$ & $\begin{array}{l}\text { Time } \\
\text { (hour) (E) }\end{array}$ & $\begin{array}{l}\text { Extracellular flavins } \\
(\mathrm{mg} / \mathrm{L}) \text { 120-hour }\end{array}$ & $\begin{array}{l}\text { Total flavins } \\
(\mathrm{mg} / \mathrm{L}) \text { 120-hour }\end{array}$ & $\begin{array}{l}\begin{array}{l}\text { Percent } \\
\text { excretion }\end{array} \\
\end{array}$ \\
\hline 1 & 0 & 0 & 0 & 0 & 24 & $57.92 \pm 0.34$ & $118.35 \pm 5.85$ & $49.07 \pm 2.13$ \\
\hline 2 & 1 & 0 & 0 & 0 & 0 & $66.49 \pm 1.22$ & $128.95 \pm 1.10$ & $51.58 \pm 1.37$ \\
\hline 3 & 0 & 2.5 & 0 & 0 & 0 & $56.4 \pm 1.170$ & $121.27 \pm 3.41$ & $46.52 \pm 0.32$ \\
\hline 4 & 1 & 2.5 & 0 & 0 & 24 & $71.12 \pm 1.95$ & $137.60 \pm 0.24$ & $51.71 \pm 1.33$ \\
\hline 5 & 0 & 0 & 2.5 & 0 & 0 & $64.30 \pm 0.97$ & $121.64 \pm 2.8$ & $52.87 \pm 0.40$ \\
\hline 6 & 1 & 0 & 2.5 & 0 & 24 & $75.41 \pm 0.88$ & $145.16 \pm 3.66$ & $51.96 \pm 0.69$ \\
\hline 7 & 0 & 2.5 & 2.5 & 0 & 24 & $67.66 \pm 1.51$ & $124.32 \pm 5.24$ & $54.50 \pm 1.09$ \\
\hline 8 & 1 & 2.5 & 2.5 & 0 & 0 & $74.59 \pm 0.73$ & $139.31 \pm 0.49$ & $53.55 \pm 0.32$ \\
\hline 9 & 0 & 0 & 0 & 25 & 0 & $52.16 \pm 1.02$ & $112.25 \pm 5.36$ & $46.61 \pm 3.16$ \\
\hline 10 & 1 & 0 & 0 & 25 & 24 & $66.30 \pm 0.93$ & $130.78 \pm 0.00$ & $50.70 \pm 0.69$ \\
\hline 11 & 0 & 2.5 & 0 & 25 & 24 & $54.21 \pm 1.02$ & $118.35 \pm 2.92$ & $45.85 \pm 1.98$ \\
\hline 12 & 1 & 2.5 & 0 & 25 & 0 & $63.08 \pm 1.02$ & $126.02 \pm 2.56$ & $50.11 \pm 1.81$ \\
\hline 13 & 0 & 0 & 2.5 & 25 & 24 & $59.23 \pm 0.97$ & $115.42 \pm 4.39$ & $51.44 \pm 2.78$ \\
\hline 14 & 1 & 0 & 2.5 & 25 & 0 & $67.81 \pm 0.58$ & $126.63 \pm 0.97$ & $53.54 \pm 0.06$ \\
\hline 15 & 0 & 2.5 & 2.5 & 25 & 0 & $58.99 \pm 0.34$ & $119.57 \pm 0.24$ & $49.30 \pm 0.39$ \\
\hline 16 & 1 & 2.5 & 2.5 & 25 & 24 & $71.81 \pm 1.56$ & $134.80 \pm 2.07$ & $53.30 \pm 2.01$ \\
\hline
\end{tabular}

\subsection{Optimizing Flavins Production with CCD}

Central composite design is shown in the Table 3 with Total flavins recorded as response. Multiple regression analysis with the experimental data gave a second-order polynomial equation as follows in terms of uncoded units:

$$
\mathrm{Y}=68.52+131.93 \mathrm{M}+29.7 \mathrm{~V}-77.72 \mathrm{M}^{2}-29.73 \mathrm{~V}^{2}+26.36 \mathrm{MV}
$$

Where, $\mathrm{Y}=$ predicated response, $\mathrm{M}=$ menadione and $\mathrm{V}=$ vitamin $\mathrm{E}$ coded values.

Table 3. Central composite design matrix with two factors - menadione and vitamin E.

\begin{tabular}{|c|c|c|c|c|}
\hline Run & Menadione $(\mu \mathrm{M})$ & Vitamin E $(\mu \mathbf{M})$ & Experimented Total flavins (mg/L) 120-hour & Predicted value \\
\hline 1 & 0.50 & 0.50 & 134.80 & 129.80 \\
\hline 2 & 1.20 & 0.50 & 143.09 & 138.89 \\
\hline 3 & 0.50 & 1.20 & 128.71 & 128.00 \\
\hline 4 & 1.20 & 1.20 & 151.13 & 150.00 \\
\hline 5 & 0.36 & 0.85 & 120.42 & 119.44 \\
\hline 6 & 1.34 & 0.85 & 136.75 & 141.42 \\
\hline 8 & 0.85 & 1.34 & 146.99 & 146.21 \\
\hline 9 & 0.85 & 0.85 & 151.13 & 149.47 \\
\hline 10 & 0.85 & 0.85 & 153.08 & 149.47 \\
\hline 11 & 0.85 & 0.85 & 146.25 & 149.47 \\
\hline 12 & 0.85 & 0.85 & 148.20 & 149.47 \\
\hline 13 & 0.85 & 0.85 & 148.69 & 149.47 \\
\hline 14 & 0.50 & 0.50 & 131.63 & 129.80 \\
\hline 16 & 0.50 & 1.20 & 123.10 & 128.00 \\
\hline 17 & 1.20 & 1.20 & 148.69 & 150.00 \\
\hline 18 & 0.36 & 0.85 & 117.98 & 119.44 \\
\hline 19 & 1.34 & 0.85 & 139.92 & 141.42 \\
\hline 20 & 0.85 & 0.36 & 133.34 & 139.63 \\
\hline 21 & 0.85 & 1.34 & 149.91 & 146.21 \\
\hline
\end{tabular}

ANOVA test (analysis of variance) was carried out for determining the optimal value. Goodness of fit results obtained from ANOVA test indicated that $89.8 \%$ could be explained by response model. Thus, the obtained model showed fair correlation between the experimental and predicted values with further improvement in $\mathrm{R}^{2}-$ value of
0.90. The results from ANOVA test is shown in Table 4 indicating effect of individual factor as well as interaction are significant for flavins production. Contour plots and response-surface plots derived from CCD revealed optimal concentration of supplements for flavins production centered near $1.09 \mu \mathrm{M}$ menadione and $1.12 \mu \mathrm{M}$ vitamin $\mathrm{E}$ (Figure 2 ). 


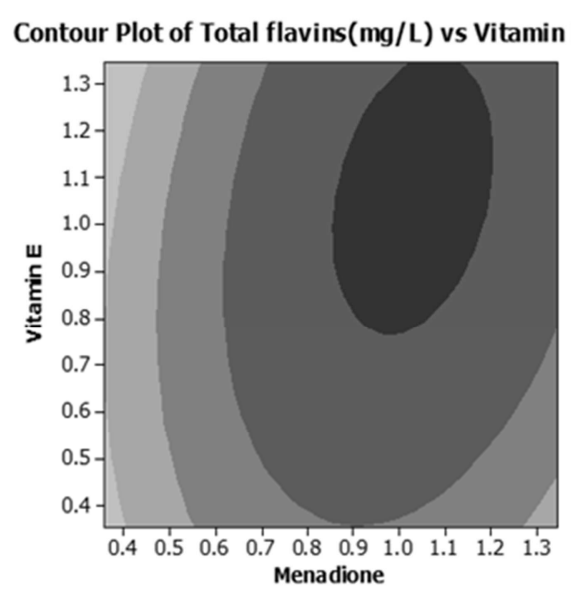

A)
Surface Plot of Total flavins(mg/L) vs Vitamin E, Menadione
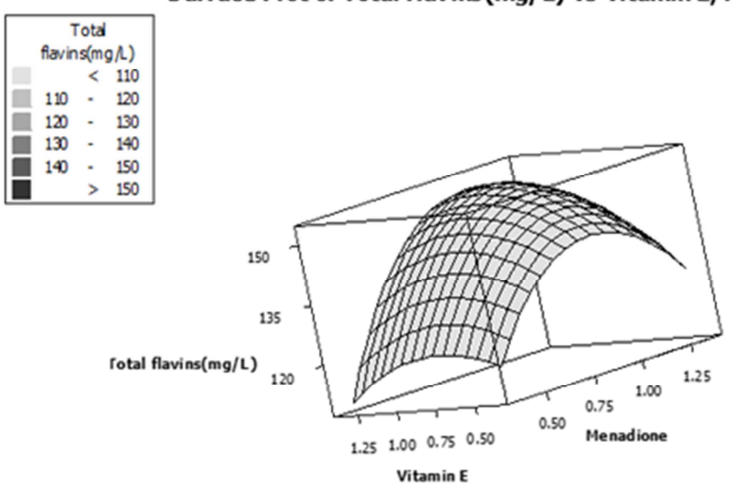

B)

Figure 2. Central composite design for quantification of optimum levels of supplements. A.) Contour plot of total flavins production as a function of menadione and vitamin E. B.) Response surface plot of total flavins production as a function of menadione and vitamin $E$.

Table 4. Regression analysis and Analysis of variance (ANOVA) test for the model obtained with CCD design for total flavins production.

\begin{tabular}{lllllll}
\hline Source & Degree of freedom & Sum of squares & Mean square & F-value & & P-value \\
\hline Regression & 5 & 2455.8 & 491.16 & 35.28 & Significance & $<0.001$ \\
Linear & 2 & 1052.62 & 526.31 & 38.8 & $<0.001$ \\
Square & 2 & 1319.78 & 659.89 & 47.4 & $<0.001$ \\
Interaction & 1 & 83.4 & 83.4 & 5.99 & Significant & Significant \\
\hline
\end{tabular}

\subsection{Time Course Analysis of Flavins Production with Optimized Oxidative Stress Modulators}

A time course analysis with optimized concentration of menadione plus vitamin $\mathrm{E}$ was followed against control (Figure 3). Menadione and vitamin E were supplemented after 24-hour of culture inoculation during mid-log phase. Significantly higher residual glucose and decrease in biomass production was observed at 48-hour of total incubation. Higher residual glucose (1.81-fold) was observed in treated $(1.47 \pm 0.18 \mathrm{~g} / \mathrm{L}) \mathrm{Vs}$ control $(0.86 \pm 0.10 \mathrm{~g} / \mathrm{L})$, Pattern of biomass production and auto-lysis were different in the treated cells (Figure 3A). Mycelia in untreated flasks showed rapid lysis from 72-hour onward. But treated mycelia were stable even up to 120-hour without undergoing autolysis unlike the control.

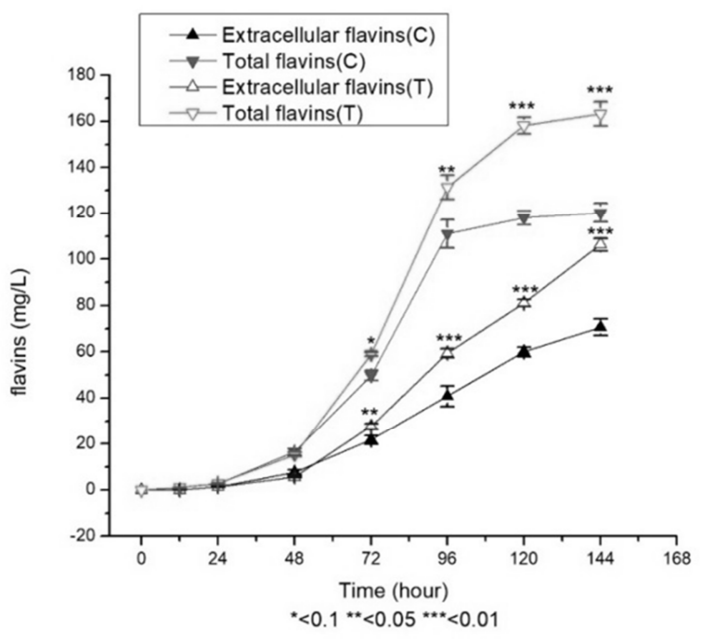

(A)

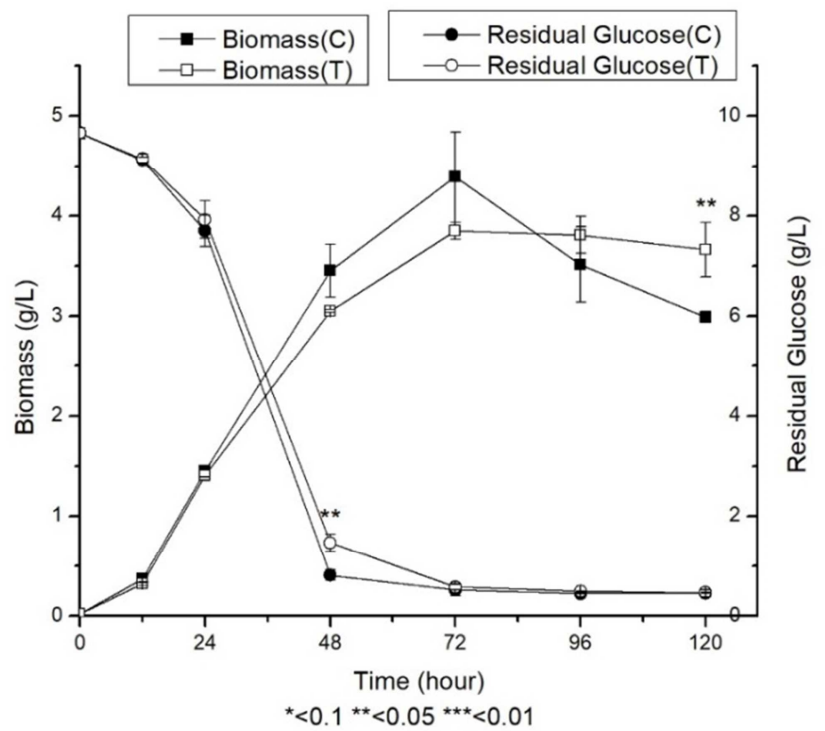

(B)

Figure 3. Time course analysis of flavins production by E.ashbyi (A) biomass production and residual glucose (B) extracellular flavins and total flavins production. Treated (T) - $1.09 \mu \mathrm{M}$ menadione and $1.12 \mu \mathrm{M}$ vitamin $E$ supplemented at 24-hour, Control (C) - Untreated culture. p-value $*<0.1$, $* *<0.05, * * *<0.01$.

Extracellular flavins production was significantly increase by 1.35 -fold and total flavins by 1.34 -fold on 120-hour of incubation (Figure 3B). Thus, with menadione and vitamin $\mathrm{E}$ combination, higher flavins production was achieved compared to menadione treatment alone (1.15-fold). Vitamin $\mathrm{E}$ could act as pro-oxidant at low levels and induce 
membrane peroxidation which resulted in increased extracellular flavins. Membrane peroxidation has been reported to affect membrane fluidty and transport earlier [22]. Similar pro-oxidant activity of vitamin E at lower levels of 1$5 \mu \mathrm{M}$ was reported to increase extracellular riboflavin by 1.70 -fold $(56.2 \pm 3.6 \mathrm{mg} / \mathrm{L}$ in control Vs. $95.8 \pm 13.8 \mathrm{mg} / \mathrm{L}$ in $2.5 \mu \mathrm{M}$ vitamin E treatment) in A.gossypii [11].

HPLC analysis of samples indicated significant increase in FAD, the end product of riboflavin biosynthetic pathway. Increase of 2.18-fold in total FAD was observed at 120-hour of incubation (Table 5). Major increase in FAD production suggested that correlation between stress and flavins metabolism is linked through FAD level regulation. Interestingly, flavins production was induced most significantly by superoxide radical generator menadione. Superoxide radical induced glutathione metabolism could have direct corelation with flavins metabolism which was studied further.

\subsection{Effect on Glutathione Metabolism}

As glutathione is a key regulator of oxidative stress [23] levels of reduced (GSH) and oxidized (GSSG) glutathione were measured in the cell free extract. At 72-hour incubation GSH was significantly decreased by 1.42 -fold and GSSG was increased by 1.35 -fold causing significant decrease in GSH/GSSG ratio ( $10.27 \pm 0.74$ Vs. $5.41 \pm 0.85$ for control Vs. treated) (Table 5). Activity of glutathione reductase was also significantly increased by 2.34 -fold at 72 -hour and 1.64 -fold at 120-hour incubation suggesting cells have sensed oxidative stress and increased the production of counter response enzyme (Table 5). Though activity of glutathione reductase was increased, GSH/GSSG ratio was lower than control indicating high production of ROS in chain reactions.

Table 5. Effect of menadione and vitamin E supplementation on glutathione and related enzymes.

\begin{tabular}{llll}
\hline \multirow{2}{*}{ Parameters } & Control & \multicolumn{2}{c}{ Treated cells } \\
\cline { 2 - 4 } & 72-hour & 120-hour & 72-hour \\
\hline Total FAD $(\mathrm{mg} / \mathrm{L})$ & $0.94 \pm 0.01$ & $13.63 \pm 0.79$ & $2.36 \pm 0.65^{*}$ \\
GSH $(\mathrm{mmoles} / \mathrm{mg})$ & $242.99 \pm 7.88$ & $258.88 \pm 36.43$ & $171.36 \pm 15.28^{*}$ \\
GSSG $(\mathrm{mmoles} / \mathrm{mg})$ & $23.79 \pm 1.91$ & $30.63 \pm 5.25$ & $32.34 \pm 5.17$ \\
GSH/GSSG & $10.27 \pm 0.74$ & $8.56 \pm 1.05$ & $5.41 \pm 0.85^{*}$ \\
GSH reductase $(\mu \mathrm{U} / \mathrm{mg})$ & $45.53 \pm 2.62$ & $71.79 \pm 7.19$ & $106.44 \pm 12.47 *$ \\
FAD synthetase $(\mu \mathrm{U} / \mathrm{mg})$ & $25.33 \pm 2.24$ & $76.85 \pm 10.44$ & $54.96 \pm 6.15^{*}$ \\
\hline
\end{tabular}

Control $=$ Untreated culture, $*=$ p-value $<0.05$.

FAD a coenzyme for glutathione reductase which plays a key role in conversion of oxidized glutathione to its reduced form, which is the active form reacting with ROS [19]. Concomitant increase in glutathione reductase enzyme activity and FAD levels suggest a dynamic link between oxidative stress and flavins production in E.ashbyi. Direct response of oxidative stress was measured through estimation of ROS by DCFDA conversion which was increased by 1.91 fold in mycelium treated for 6-hour $(3.51 \pm 0.73 \mathrm{nmol} / \mathrm{mg}$ of protein) vs. control $(1.83 \pm 0.12 \mathrm{nmol} / \mathrm{mg}$ of protein).

\subsection{Effect on FAD Synthetase Enzyme Activity}

FAD is a coenzyme for many of the stress response and metabolic enzymes. Promoter study of FAD1 gene (encoding for FAD synthetase enzyme) of closely related yeast Saccharomyces cerevisiae with YEASTRACT toolbox showed presence of binding sites for stress response transcription factors - three YAP1 binding sites at -418, -552 and -695 positions and $M S N 2$ binding site at -8 . Further, FAD synthetase activity was studied in 72-hour and 120-hour grown culture of E.ashbyi to analyze its role as stress response enzyme. Interestingly, FAD synthetase activity was increased by 2.16 -fold in 72-hour grown culture and 3.06fold in 120-hour grown culture (Table 5).

De novo synthesis of FAD through fermentative route provides a great advantage over enzymatic or microbial biotransformation which uses expensive intermediates such as FMN and ATP. De novo synthesis of FAD was reported in yeast and fungi so far while biotransformation was reported mainly in bacteria. Metabolic engineering of Candida famata with overexpression of FMN1 and FAD1 genes, resulted in $70 \mathrm{mg} / \mathrm{L}$ FAD titer on YPD broth compared to $4 \mathrm{mg} / \mathrm{L}$ in WT and media optimization through Central Composite Design increased it $451 \mathrm{mg} / \mathrm{L}$ FAD [2]. Overexpression of FMN1 gene in A.gossypii increased FAD production by 14.02 -fold with maximum titer of $86.5 \mathrm{mg} / \mathrm{L}$ [16]. Nearly $194 \mathrm{mg} / \mathrm{L}$ FAD was obtained in earlier literature with E.ashbyi with culture and complex media components optimization [24]. In the present study, maximum FAD titer of $29.73 \pm 2.22 \mathrm{mg} / \mathrm{L}$ was obtained with YM broth with wild type strain. Flavins production was nearly 16.5 -fold higher with complex media components like molasses and peanut seed cake compared to simple sugars like glucose [25]. Further studies can be conducted to improve FAD titer through media and culture conditions optimization using complex medium components along with oxidative stress supplements.

YAP1 transcription factor was reported to regulate glutathione reductase (GSR) gene in Saccharomyces cerevisiae [26] and it is also reported to regulate riboflavin metabolism (RIB) genes in Ashbya gossypii [14]. Putative role of Yap-1 in FAD1 gene regulation can be studied further. Moreover, presence of binding sites for stress response transcription factors like $Y A P 1$ and MSN2 in FAD1 gene promoter indicated FAD synthetase itself as one of the major putative stress response enzymes and warrants further studies on FAD1 gene expression under oxidative stress. Present experimental findings suggest oxidative stress increase flavins in particular FAD synthesis by upregulating FAD 
synthetase and increased FAD helps in glutathione mediated oxidative stress response. Accordingly, putative mechanism of FAD synthetase as oxidative response enzyme is shown in Figure 4.

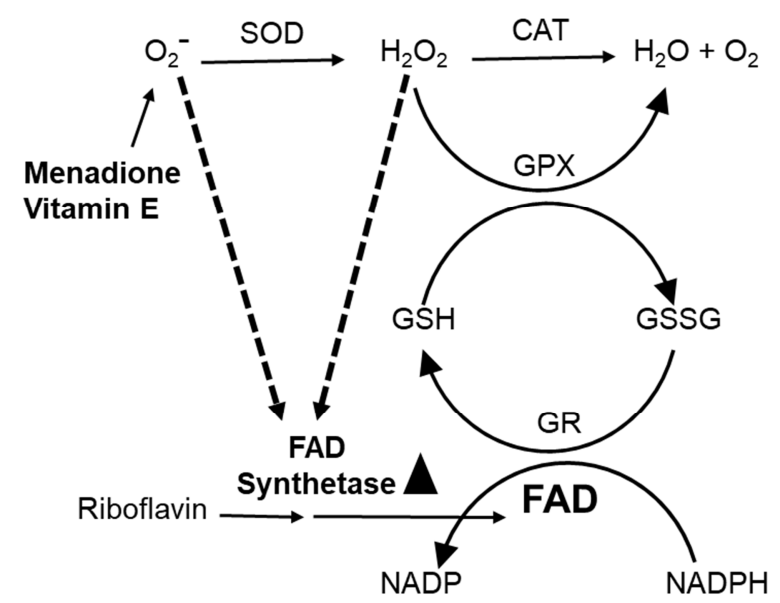

Figure 4. Putative mechanism for enhanced FAD production upon oxidative stress. Compiled from Kavitha and Chandra, 2014 [13].

Flavins production in response to oxidative stress can be of evolutionary as well as ecological importance. Being a plant parasite, E.ashbyi may encounter an oxidative burst as a defensive action mechanism developed by plants in response to microbial invasion [27]. Upregulation of riboflavin and FAD production can aid fungal own defense system in quickly scavenging the free radicals. Increased flux towards FAD synthesis can be due to its direct role as electron mediator in stress response enzymatic reactions.

\section{Conclusion}

This is a first report on statistical optimization of oxidative stress modulators for increasing flavins production in E.ashbyi. Menadione and vitamin E combination at low levels enhanced flavins production by 1.34 -fold against untreated control and 1.15-fold against menadione treatment alone. FAD production was significantly increased with these supplements' combination by 2.18 -fold over untreated control. For the first time present study proved that among the flavins, FAD levels increased majorly upon oxidative stress. FAD synthetase specific enzyme activity was also concurrently increased with stress response enzyme glutathione reductase. This finding suggested that the FAD synthetase can also be a stress response enzyme. As FAD is a coenzyme for several redox reactions, increased flux towards FAD can help in oxidative stress response including glutathione mediated response as observed here.

\section{Acknowledgements}

We gratefully acknowledge Department of Biotechnology, Government of India for funding this project (BT/PR5974/PID/6/674/2012).

\section{References}

[1] Colibus L. and Mattevi A. (2006) New frontiers in structural flavoenzymology, Curr. Opin. Struct. Biol. 16: 722-728.

[2] Yatsyshyn V., Fedorovych D. and Sibirny A. (2014) Metabolic and bioprocess engineering of the yeast Candida famata for FAD production, J. Ind. Microbiol. Biotechnol. 41: 823-835.

[3] Gonzalez-Cabo P., Ros S. and Palau F. (2014) Flavin adenine dinucleotide rescues the phenotype of frataxin deficiency, PLoS One 5: 1-10.

[4] Huang Y., Liu S., Yen C., et. al. (2009) Thapsigargin and flavin adenine dinucleotide ex vivo treatment rescues trafficking-defective gp91phox in chronic granulomatous disease leukocytes, Free Radic. Biol. Med. 47: 932-940.

[5] Ashby S. and Nowell W. (1926) The Fungi of Stigmatomycosis, Ann. Bot. 40: 69-84.

[6] Kato T. and Park E. (2012) Riboflavin production by Ashbya gossypii, Biotechnol. Lett. 34: 611-618.

[7] Abbas C. and Sibirny A. (2011) Genetic Control of Biosynthesis and Transport of Riboflavin and Flavin Nucleotides and Construction of Robust Biotechnological Producers, Microbiol. Mol. Biol. Rev. 75: 321-360.

[8] Ozbas T. and Kutsal T. (1986) Comparative study of riboflavin production from two microorganisms: Eremothecium ashbyi and Ashbya gossypii, Enzyme Microb. Technol. 8: 593-596.

[9] Protchenko O., Boretsky B., Romanyuk T., et. al. (2000) Oversynthesis of riboflavin by yeast Pichia guilliermondii in response to oxidative stress, Ukr. Biokhimichnyi Zhurnal 72: $19-23$.

[10] Schlösser T., Wiesenburg A., Gätgens C., et. al. (2007) Growth stress triggers riboflavin overproduction in Ashbya gossypii, Appl. Microbiol. Biotechnol. 76: 569-578.

[11] Kavitha S. and Chandra T. S. (2009) Effect of vitamin E and menadione supplementation on riboflavin production and stress parameters in Ashbya gossypii, Process Biochem. 44: 934-938.

[12] Stahmann K., Arst H., Althofer H. et. al. (2001) Riboflavin, overproduced during sporulation of Ashbya gossypii, protects its hyaline spores against ultraviolet light, Environ. Microbiol. 3: $545-550$.

[13] Kavitha S. and Chandra T. S. (2014) Oxidative Stress Protection and Glutathione Metabolism in Response to Hydrogen Peroxide and Menadione in Riboflavinogenic Fungus Ashbya gossypii, Appl. Biochem. Biotechnol. 174: 2307-2325.

[14] Walther A. and Wendland J. (2012) Yap1-dependent oxidative stress response provides a link to riboflavin production in Ashbya gossypii, Fungal Genet. Biol. 49: 697-707.

[15] Ashoori M. and Saedisomeolia A. (2014) Riboflavin (vitamin B2) and oxidative stress: a review, Br. J. Nutr. 111: 1985-1991.

[16] Patel M. and Chnadra T. S. (2019) Metabolic engineering of Ashbya gossypii for enhanced FAD production through promoter replacement of FMN1 gene, Enzyme Microb. Technol. 133. 
[17] Ayar-Kayali H., Ozer N. and Tarhan L. (2002) Intracellular superoxide dismutase, catalase, and glutathione peroxidase activities and membrane lipid peroxide levels in Fusarium acuminatum upon environmental changes in a defined medium, Arch. Biochem. Biophys. 400: 265-272.

[18] Tietze F. (1969) Enzymic method for quantitative determination of nanogram amounts of total and oxidized glutathione: Applications to mammalian blood and other tissues, Anal. Biochem. 27: 502-522.

[19] Mavis R. and Stellwagen E. (1968) Purification and Subunit of Glutathione Reductase from Bakers Yeast, J. Biol. Chem. 243: 809-814.

[20] Rastogi R., Singh S., Hader D., et. al. (2010) Detection of reactive oxygen species (ROS) by the oxidant-sensing probe 2',7'-dichlorodihydrofluorescein diacetate in the cyanobacterium Anabaena variabilis PCC 7937, Biochem. Biophys. Res. Commun. 397: 603-607.

[21] Orth A., Sfarra A., Pell E., et. al. (1993) Assessing the Involvement of Free Radicals in Fungicide Toxicity Using $\alpha$ Tocopherol Analogs, Pestic. Biochem. Physiol. 47: 134-141.

[22] Wong-Ekkabut J., Xu Z., Triampo W., et. al. (2007) Effect of lipid peroxidation on the properties of lipid bilayers: a molecular dynamics study, Biophys. J. 93: 4225-36.

[23] Grant C. (2001) Role of the glutathione/glutaredoxin and thioredoxin systems in yeast growth and response to stress conditions, Mol. Microbiol. 39: 533-541.

[24] Tsukihara K., Minoura K. and Izumiya M. (1960) Studies on the industrial production of flavin-adenine dinucleotide. Preparation of mycelium of Eremothecium ashbyi as a raw material containing flavin-adenine dinucleotide, J. Vitaminol. (Kyoto). 6: 68-76.

[25] Lim S., Choi J. and Park E. (2001) Microbial Production of Riboflavin Using Riboflavin Overproducers Ashbya gossypii, Bacillus subtilis, and Candida famata: An Overview, Biotechnol. Bioprocess Eng. 6: 75-88.

[26] Grant C., Collinson L., Roe J. et. al. (1996) Yeast glutathione reductase is required for protection against oxidative stress and is a target gene for $y A P-1$ transcriptional regulation, Mol. Microbiol. 21: 171-179.

[27] Wojtaszek P. (1997) Oxidative burst: an early plant response to pathogen infection, Biochem. J. 322: 681-92. 